\title{
Amphoteron mántin te agathòn kaì dorì máchestai: reelaboraciones poéticas y (re)performance en O. 6. 12-18 y CEG 519
}

\author{
Amphóteron mántin te agathòn kai dori máchestai: poetic reworkings and (re)performance in O. 6. 12-18 and CEG 519
}

\author{
Caterina Anush Stripeikis \\ Universidad de Buenos Aires, Argentina \\ caterina.stripeikis@gmail.com \\ https://orcid.org/0000-0001-7614-9694
}

\begin{abstract}
Resumen:
El objetivo del presente trabajo es estudiar los modos de adaptación y reelaboración de la frase a $\mu \phi o ́ \tau \varepsilon p o \nu ~ \mu \alpha \dot{\nu} \tau \tau \nu \tau \varepsilon \dot{\alpha} \gamma \alpha \theta \dot{o} \nu$ kaì $\delta \circ p i ~ \mu \alpha ́ \chi \varepsilon \varepsilon \sigma \theta a$ ("a la vez buen adivino y bueno para combatir con la lanza"), cuya primera aparición parece haber tenido lugar en la obra perteneciente a los cantos cíclicos conocida como la Tebaida. Para acometer este estudio se tomarán en cuenta dos producciones posteriores en las que dicha frase resurge: la Olímpica 6 de Píndaro y el epigrama número 519 de la colección de Carmina Epigraphica Graeca (CEG) de Hansen. Se analizará la adaptación poética del sintagma a los objetivos y procedimientos compositivos característicos de ambas producciones en el marco de un contexto histórico y socio-cultural más amplio. Asimismo,

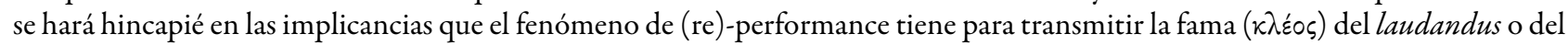
difunto a través del empleo de dicha frase.
\end{abstract}

Palabras ClaVe: Epinicio, Epigrama, Reelaboración, Performance.

\section{Abstract:}

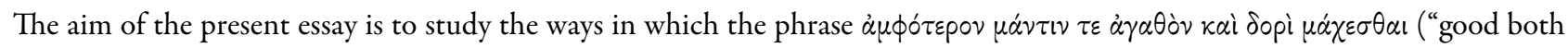
as a seer and as a fighter with the spear"), apparently first attested in the cyclic Thebaid, is adapted and reworked in two poetic productions. The first is Pindar's Olympian 6 and the second is epigram number 519 from the Carmina Epigraphica Graeca (CEG) edited by Hansen. I will analyze the poetic adaptation of the phrase to the goals and compositional procedures displayed by both productions, considering, at the same time, the wider historical and socio-cultural frame that surrounds them. I will also highlight the importance of the (re)-performance phenomenon in order to transmit the fame ( $\kappa \lambda \varepsilon \dot{o s})$ of the laudandus or the deceased through the deployment of the phrase mentioned above.

KeYwORDs: Epinikion, Epigram, Reworking, Performance.

\section{INTRODUCCIÓN}

Promediando el primer epodo de la Olímpica 6, el poeta dice del laudandus Hagesias que este es "a la vez

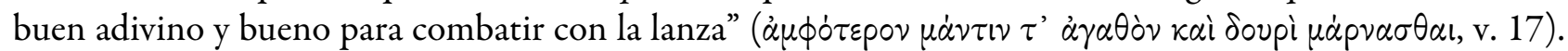
La aparición de la frase ha motivado el siguiente comentario de uno de los escoliastas al texto pindárico: $\delta$

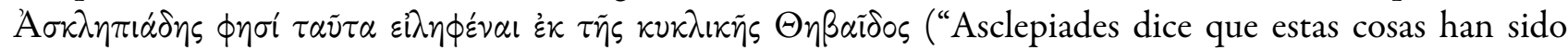
tomadas de la épica cíclica la Tebaida”, $\Sigma$ 6. 26). La apreciación del escoliasta es bastante razonable, si se considera que en el pasaje Píndaro articula el elogio de Hagesias a partir de la mención del adivino Anfiarao, una de las figuras protagónicas en esta épica. Asimismo, esta afirmación ha sido tomada de modo literal por algunos editores de la fragmentaria Tebaida, que no han dudado en suplementar la frase textualmente, cambiando, por razones métricas, el infinitivo $\mu \dot{\alpha} \rho \nu \alpha \sigma \theta \alpha \iota$ que figura en la Olimpica por su coetáneo $\mu \alpha ́ \chi \varepsilon \sigma \theta \alpha \iota$ o $\mu \alpha \chi \eta \tau \eta ́ v$, más apropiado para un esquema hexamétrico (cfr. F7 Davies, F1 Bernabé). 
Aquí se entra, indefectiblemente, en un terreno especulativo, puesto que el comentario del escoliasta podría estar apuntando simplemente a una coincidencia de contenido temático entre el pasaje de la Olímpica 6 y la Tebaida. En efecto, el empleo del sustantivo $\tau \alpha \tilde{\tau} \tau \alpha$ ("estas cosas") tiñe al escolio de un alto grado de ambigüedad. En consonancia con este razonamiento, ya Wilamowitz (1886, pp. 163-164, n. 4) y Robert (1915, pp. 247-251) planteaban que el pasaje para llenar el fragmento de la Tebaida debía buscarse no en

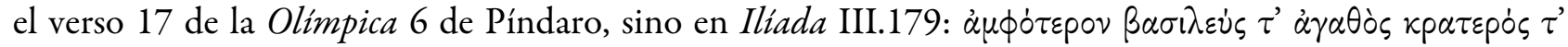

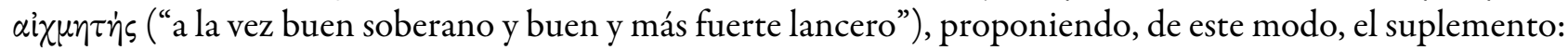

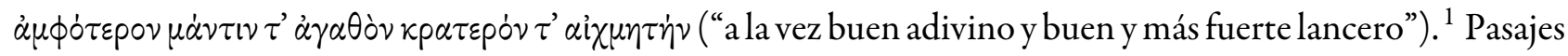
semejantes a Olimpica 6.17 también figuran en Hesíodo, fr. 25.37 M-W, Esquilo, Siete contra Tebas 568-569 y Sófocles, Edipo en Colono 1313-1314, estos últimos tres referidos al adivino Anfiarao.

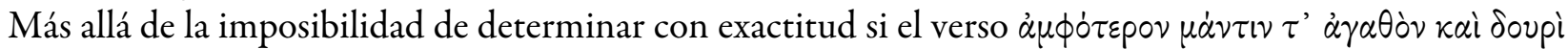
$\mu \dot{\alpha} \rho v \alpha \sigma \theta \alpha \mathrm{l}$ ("a la vez buen adivino y bueno para combatir con la lanza”) representa una invención pindárica o una copia casi textual de la Tebaida, resulta indiscutible que este se encuentra dialogando, en líneas generales, con una tradición épica precedente, también perceptible en el drama ático. Un siglo más tarde, el sintagma resurge de modo casi idéntico en un epigrama ático dedicado al difunto adivino Cleóbulo, hijo de Glauco.

El objetivo del presente trabajo es, pues, estudiar los modos de adaptación y reelaboración de la frase

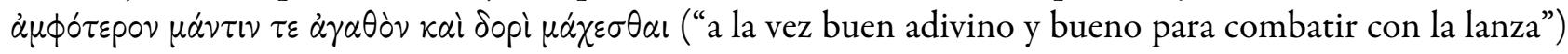
en la Olimpica 6 de Píndaro y en $C E G 519$, haciendo especial hincapié en la funcionalidad de la frase para cumplir los objetivos e iluminar los procedimientos compositivos característicos de ambas producciones. El análisis se complementará apelando al contexto histórico y socio-cultural más amplio que se desprende tanto de la inscripción como de la oda y a la noción de (re)-performance, medio que permite vehiculizar la fama

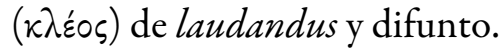

\section{Olímpica 6. 12-18 y CEG 519: Construyendo el Elogio mántico-BÉlico de Hagesias y Cleóbulo.}

La Olímpica 6 de Píndaro fue compuesta para celebrar la victoria del atleta Hagesias en la carrera de mulas, bien en el año 472 a.C. o 468 a.C. ${ }^{2}$ El homenajeado parece haber sido, a juzgar por el contenido de la oda, una figura cercana al círculo del tirano siracusano Hierón con una trayectoria de vida particularmente interesante. Píndaro lo caracteriza como administrador del altar oracular de Zeus en Pisa $(\beta \omega \mu \tilde{\omega} \tau \varepsilon \mu \alpha \nu \tau \varepsilon i \omega$ $\tau \alpha \mu i \alpha \varsigma \Delta ı \iota_{\zeta} \xi \dot{\nu} \prod i \sigma \alpha$, v. 5), profesión hereditaria que le corresponde en tanto descendiente por vía paterna del

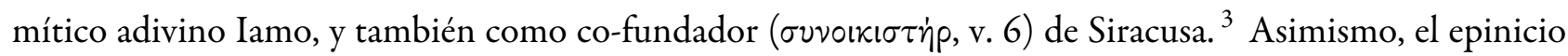
hace mención de su ascendencia masculina por parte materna, oriunda de Estínfalo en Arcadia (v. 77), circunstancia que permite completar la descripción del vencedor Hagesias como un individuo de doble "nacionalidad", estinfalia y siracusana (vv. 97-102).

El pasaje objeto de análisis ocupa los versos 12-18 de la oda y se enmarca en un pequeño excursus mitológico relacionado con las facultades adivinatorias de Hagesias:

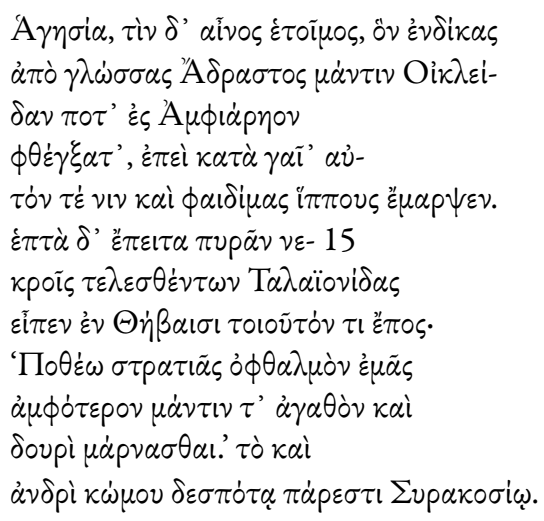




\begin{abstract}
Hagesias, a ti te está dispuesto el elogio verdadero que con justa lengua antaño dijo Adrasto sobre el adivino Anfiarao, hijo de Oícles, después de que la tierra lo hundió a él y a sus brillantes yeguas. Luego de consumidas siete piras para los cadáveres, el hijo de Tálao pronunció en Tebas este discurso: "Extraño al ojo de mi ejército, a la vez buen adivino y bueno para combatir con la lanza.

"Esto también conviene al varón siracusano, soberano del cortejo. ${ }^{4}$
\end{abstract}

El movimiento general que presenta el pasaje es conocido por todos los estudiosos de Píndaro: un mito del acervo tradicional (épico) griego aparece resignificado para articular el elogio del laudandus. ${ }^{5}$ En esta oportunidad, sin embargo, dicha resignificación tiene lugar, principalmente, a partir de la inclusión de un discurso directo que Píndaro adapta o compone (cfr. Introducción) para aunar las facultades heroicas de Anfiarao y Hagesias. La inclusión de dicho discurso se da en el marco de una estructuración compleja que busca generar expectativa en la audiencia del epinicio y desplegar la maestría compositiva del poeta. En efecto, la frase aparece solo luego de que Píndaro haya clarificado quiénes fueron su creador y receptor originales (Adrasto-Anfiarao) y de que haya explicitado el contexto en el que esta fue pronunciada por primera vez (hundimiento de Anfiarao- quema de los cadáveres de los guerreros fallecidos en el enfrentamiento). Las instancias previas generan una sensación de crescendo que culmina, finalmente, con las esperadas palabras del hijo de Tálao. Asimismo, y tal como ha demostrado Adorjáni (2014, p. 136), la primera parte del pasaje (vv. 12-16) se estructura en términos de una pequeña Ringkomposition que se abre y se cierra con la mención de

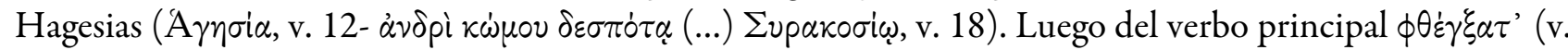
14), el pasaje retrocede al pasado cercano a partir del subordinante temporal $\dot{\varepsilon} \pi \varepsilon \dot{~(v . ~ 14) ~ p a r a ~ n a r r a r ~ l a ~}$ desaparición de Anfiarao bajo la tierra. A continuación, el relato sigue desplegándose de forma lineal (aún en el pasado), esta vez empleando el adverbio हैं $\varepsilon \iota \tau \alpha$ (v. 15) para dar cuenta de la quema de los cadáveres. Es justamente en este punto en el que el texto nos lleva, una vez más, a la primera situación enunciativa planteada

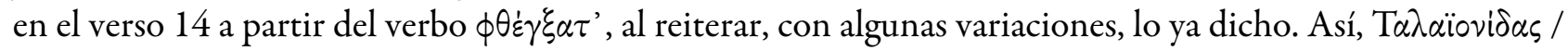

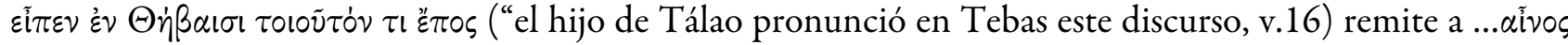

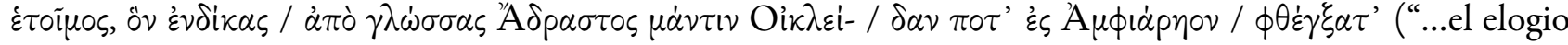
verdadero que con justa lengua antaño dijo Adrasto sobre el adivino Anfiarao, hijo de Oícles, vv. 13-14).

Como ya se mencionó, recién en el verso 17, luego de la segunda introducción al discurso directo de

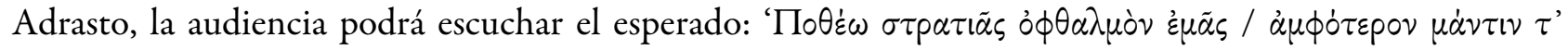

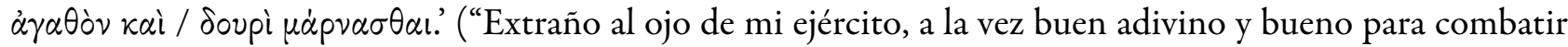
con la lanza"). La recepción de tan anticipado discurso le presenta, asimismo, algunas sorpresas adicionales al

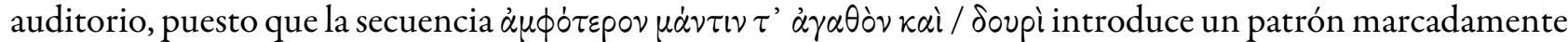
hexamétrico en el esquema dáctilo-epitrito general de la oda. En palabras de Hutchinson (2001): "at one point in the epode (2-3) the usual patterns are modified for so substantial a sequence that the listener is almost thrown off course. This is more notable than a simple extension or abridgement (...). It must particularly underline the point of first occurrence, the short and significant speech of Adrastus" (p. 375). Acaso la existencia de dicho patrón hexamétrico, roto por la presencia del infinitivo $\mu \dot{\alpha} \rho v \alpha \sigma \theta \alpha \mathrm{l}$, pueda constituir una evidencia de que Píndaro adaptó el pasaje a partir del contenido de la Tebaida (cfr. Introducción).

Aproximadamente un siglo después de la composición y performance de la Olímpica 6, se erigió una estela funeraria en las inmediaciones del distrito de Acarnas (Ática) con la siguiente inscripción:

(i, supra aquilam anaglyphi litteris maioribus incisus)

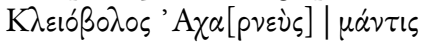

(ii, infra anaglyphon incisus)

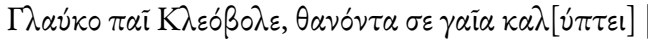

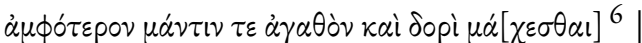

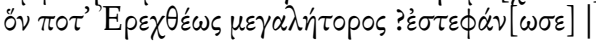

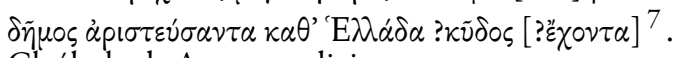

Cleóbulo de Acarnas, adivino 
Cleóbulo, hijo de Glauco, la tierra te oculta muerto,

a la vez buen adivino y bueno para combatir con la lanza,

al que antaño el pueblo de Erecteo de gran corazón ¿̨coronó?,

siendo el mejor por toda la Hélade, ¿obteniendo gloria?

La inscripción ha sido datada sin demasiada certeza entre el 375-350 a.C. Un terminus post quem bastante plausible para su inserción parece haber sido la guerra de Corinto que tuvo lugar entre el 395-397 a.C. En efecto, este mismo Cleóbulo, tío materno del orador Esquines, fue elogiado por él en su discurso De falsa legatione 2.78, con motivo de la heroica actuación que llevó a cabo en dicha guerra frente a Xeilón, comandante naval de los lacedemonios.

El epigrama presenta, en líneas generales, un estilo arcaizante que lo aleja de los ejemplos contemporáneos del género, emparentándolo con aquellos epigramas funerarios propios de los siglos VI-V a.C. dedicados a guerreros fallecidos en enfrentamientos bélicos. ${ }^{8}$ Dicho estilo aparece vehiculizado a partir del uso del hexámetro dactílico (ya casi completamente opacado en el siglo IV a.C. por el empleo del dístico) y por las deudas temáticas y sintácticas que el epigrama exhibe con el universo de la épica. En efecto, CEG 519 incorpora con ligeras modificaciones el verso 547 del llamado catálogo de las naves que figura en el canto 2

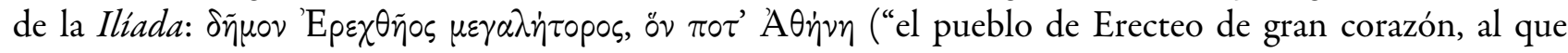

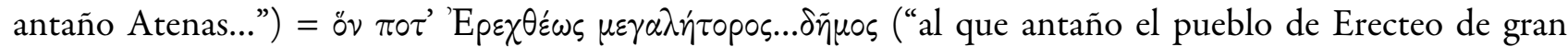
corazón...”). También el monumento que contiene el epigrama ofrece un nuevo tributo a la profesión de Cleóbulo en el marco de la tradición épica. Su bajo relieve exhibe la imagen de un águila llevando entre sus garras una serpiente, signo mántico característico de la práctica adivinatoria que remite al portento visto por el adivino Polidamante en Ilíada 12.200-207. Estos detalles permiten aseverar con un grado bastante alto de certeza que, o bien el escultor y el epigramatista, o bien la familia del difunto que encargó el complejo, estaban versados en la tradición épica precedente y buscaron emplear sus patrones de modo deliberado para exaltar la figura de Cleóbulo.

Regresando ahora a las similitudes entre la Olímpica 6 y $C E G 519$, ya se ha explicitado que la deuda del epigrama con esta Olimpica se deja sentir, en primer lugar, a partir del empleo de una frase casi idéntica con

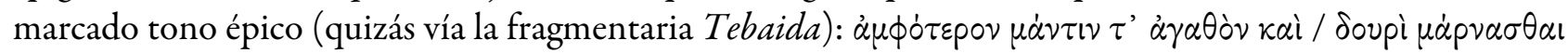

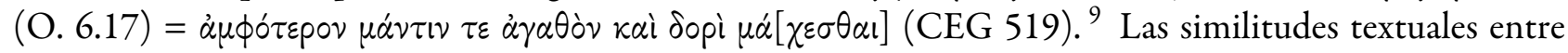
epigrama y Olimpica quizás puedan continuar afianzándose si se considera con más detenimiento el giro öv... $\pi \circ \tau \varepsilon$, presente tanto en $C E G 519$ como en Olímpica 6. 12-14. Si bien en el caso del epigrama su empleo parece derivarse del pasaje del catálogo de las naves anteriormente citado, no resulta pertinente descartar de forma tan abrupta su vínculo con la otra producción que la inscripción emula de modo más conspicuo. En

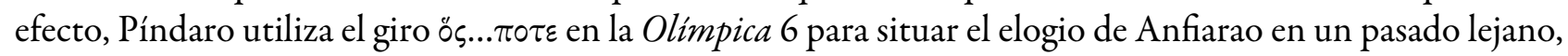
reflejando así el punto de vista de la poesía conmemorativa sobre héroes cuya existencia antecede al bic et

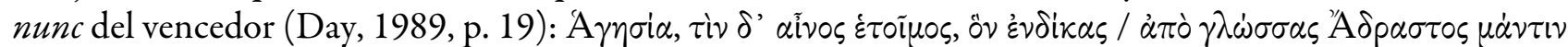

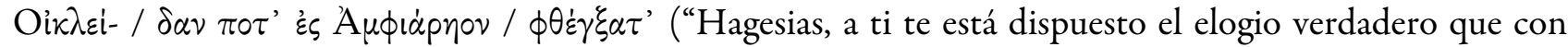
justa lengua antaño dijo Adrasto sobre el adivino Anfiarao, hijo de Oícles”, vv. 12-14). Salvando las diferencias, este mismo giro también es empleado por el epigramatista para remitir a los lectores de la inscripción a una suerte de pasado heroico-poético en el que Cleóbulo habría sido honrado con creces por el pueblo de Erecteo. En efecto, la inscripción presenta un movimiento compositivo que va desde un apóstrofe actual al difunto, más o menos informal, $\sigma \varepsilon \gamma \alpha i \alpha \kappa \alpha \lambda\left[v^{\prime} \pi \tau \varepsilon l\right]$ ("la tierra te oculta"), hasta un tiempo anterior que construye con pinceladas épico-conmemorativas la existencia del hijo de Glauco: ö $\nu$ $\pi \circ \tau^{\prime}$ 'E pueblo de Erecteo de gran corazón ¿coronó?, / siendo el mejor por toda la Hélade, ¿̇obteniendo gloria?”). La

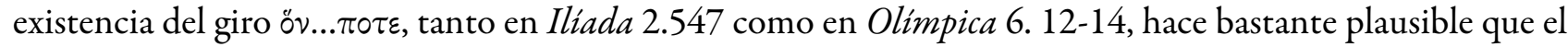
epigramatista que compuso $C E G 519$ haya tenido en cuenta las implicancias del sintagma para construir el elogio de Cleóbulo no solo a partir del precedente épico-homérico, sino también a partir de su reutilización 
pindárica, sobre todo teniendo en cuenta que tanto oda como epigrama persiguen la exaltación específica de un individuo con características y atributos análogos.

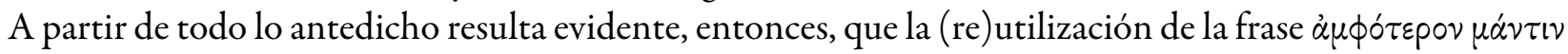

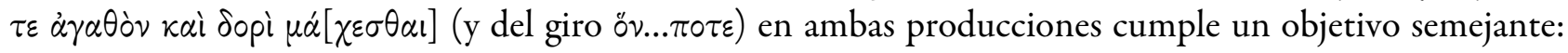
articular el elogio de Cleóbulo y de Hagesias en calidad de adivinos y combatientes bajo una dimensión épicoconmemorativa. ${ }^{10}$ En el caso de Cleóbulo, ya se sabe, a partir del testimonio transmitido por Esquines arriba mencionado, que este individuo participó en la guerra de Corinto. La actuación militar de Hagesias no resulta tan clara en el marco de la Olímpica 6, pero es dado suponer que, como miembro del círculo íntimo de Hierón, a menudo habría acompañado al tirano en sus enfrentamientos bélicos. ${ }^{11}$

La conjunción de las habilidades adivinatorias y militares empleadas en la caracterización de Cleóbulo y de Hagesias abre el interrogante acerca del rol y la función del adivino en el campo de batalla. Según Flower (2008, pp. 95-96), los adivinos no solo hacían las veces de consejeros e intérpretes de los reyes y generales en combate, a menudo sacrificando e interpretando augurios y portentos, sino que podían cumplir un rol activo también como soldados en el enfrentamiento, llegando incluso a "ganar" batallas para sus clientes. ${ }^{12}$ A juzgar por el tono general de $C E G 519$, su empleo de la frase objeto de la presente investigación y el verso

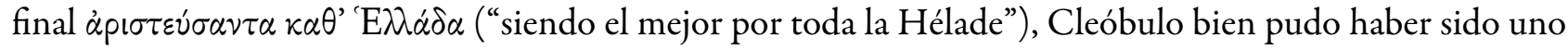
de aquellos adivinos-guerreros que tuvieron un rol activo en la procuración de la victoria. A este respecto, también es pertinente recordar el cuadro descripto por Esquines de su participación en la batalla naval contra los lacedemonios (cfr. arriba). En el caso de Hagesias, este es calificado en la oda como "ojo del

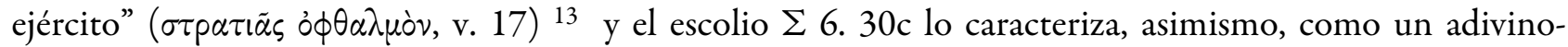

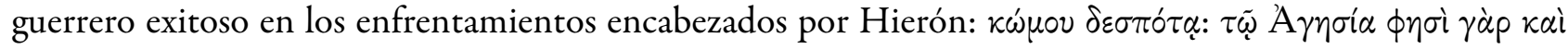

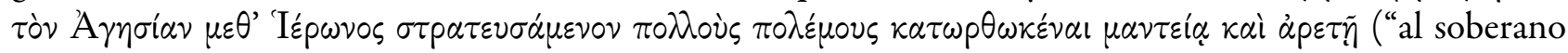
del cortejo: a Hagesias, pues también se dice que Hagesias llevó a buen término muchas batallas con la adivinación y la excelencia, combatiendo junto a Hierón”). De igual modo, el estatus mántico-bélico del laudandus se podría reforzar a partir de la consideración de la etimología de su nombre, emparentada con el verbo ä $\gamma \omega$ ("guiar, conducir"), etimología que, aunque remite de modo más directo a la maestría de Hagesias en la carrera de mulas, también puede asociarse con su desempeño en el campo de batalla. Así, Olimpica 6.12-18 y CEG 519 se enmarcan dentro de un universo cultural compartido en el que la exaltación mántico-bélica resulta un eje estructurante del elogio recibido por Cleóbulo y Hagesias.

Sin embargo, existe un punto en el que Olímpica 6.17 y CEG 519. 2 difieren notablemente. En el caso

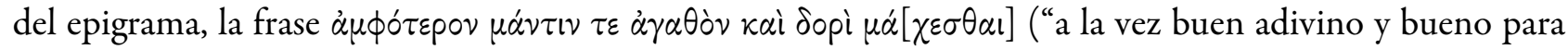
combatir con la lanza”) se inserta en una composición en la que no existe ningún impedimento para llevar el elogio de Cleóbulo a un plano hiperbólico. Se trata exclusivamente de su epigrama funerario y, en tanto tal, puede incluir frases tales como $\alpha$ p situación que presenta la Olímpica 6 es un tanto más compleja, puesto que, si bien la oda se encuentra dedicada al adivino, atleta y combatiente Hagesias, el elogio de esta figura debe balancearse allí cuidadosamente con el de su cercano superior Hierón, tirano de Siracusa. En este sentido, la exaltación excesiva del laudandus en el plano atlético y militar podría ocasionar algunos conflictos. Acaso este sea uno de los motivos por los que el poeta ha preferido enmarcar la frase objeto de la presente investigación en un pequeño excursus mitológico que permite leer tanto la figura de Hagesias como de Hierón en clave heroica. Con respecto a esta cuestión, es pertinente recordar que Píndaro pone en boca del héroe Adrasto la

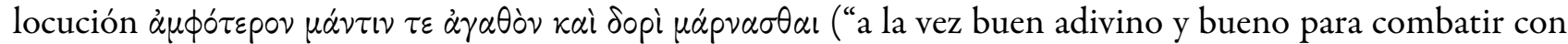
la lanza", v. 17) referida al adivino Anfiarao, y que la encabeza con el sintagma (quizás también tomado de la

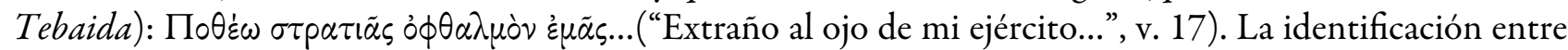
Hagesias y el paradigma mítico de Anfiarao es indiscutible. Ahora bien, ¿es posible encontrar un correlato contemporáneo al paradigma mítico de Adrasto?La asociación de este héroe con el tirano Hierón resulta la más plausible, en tanto el discurso pronunciado por Adrasto resulta significativo para ilustrar el vínculo colaborativo entre jefe y adivino-guerrero en el campo 


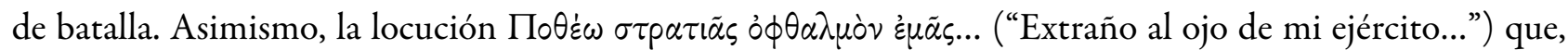
en el caso de la secuencia mítica, apunta a la desolación de Adrasto por la desaparición de Anfiarao bajo la tierra, puede trasladarse al hecho de que Hierón "echaría de menos” a Hagesias, ya porque este se tuvo que ausentar de Siracusa para competir en los juegos olímpicos, ya porque permaneció en Estínfalo un tiempo, antes de retornar a la isla. ${ }^{14}$

\section{OLÍMPICA 6. 12-18 y CEG 519: conteXtos (RE)PERFORMATIVOS Y TRANSMisión DE KLÉOS}

Otro de los objetivos del presente trabajo consiste en el estudio de las implicancias que el fenómeno de (re)performance tiene para transmitir la fama ( $\kappa \lambda \varepsilon \varepsilon$ ś) del laudandus o del difunto a través del empleo de la frase

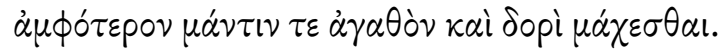

En el caso del epinicio pindárico, el término re-performance señala las múltiples ejecuciones de la oda pindárica luego de su primera instanciación. Más allá de las discusiones acerca de la modalidad y los contextos concretos de estas ejecuciones, hoy en día está fuera de duda que las odas de Píndaro no constituían piezas únicas, descartables luego de la premier, sino que resultaban composiciones susceptibles de representarse una y otra vez, por lo menos durante el período de vida del laudandus o de sus familiares y amigos cercanos. ${ }^{15} \mathrm{La}$ existencia de esta dinámica performativa entra en consonancia, por otro lado, con las ambiciones y deseos de los vencedores celebrados en las odas, que buscaban proyectar su fama y la de su familia a la posteridad y veían en la re-performance una estrategia fructífera para cumplir dicho propósito.

La re-performance no es un fenómeno único del epinicio pindárico o de géneros tales como el drama ático, en los que es fácil imaginar un despliegue más o menos impresionante de música, baile y demás parafernalia estética en cada representación. Lo cierto es que composiciones más humildes, como el epigrama aquí analizado (y los epigramas inscriptos en general), también son susceptibles al fenómeno re-performativo a través del acto de lectura. En este sentido, y a partir del trabajo de Svenbro (1988) sobre hábitos y prácticas de lectura en la Grecia arcaica y clásica, Day (2010) ha planteado de manera bastante convincente que los testimonios epigráficos despliegan una serie de estrategias para atraer la atención de sus posibles receptores y propiciar el acto de lectura. ${ }^{16}$ Así, cada transeúnte suficientemente interesado por una inscripción como para leerla en voz alta se encontraría re-presentándola a través de esta práctica y, en consecuencia, generando una vez más determinados efectos estéticos, sociales y religiosos. En el caso de $C E G 519$, inscripción en la que se articulan mecanismos laudatorios íntimamente emparentados con el elogio del vencedor pindárico Hagesias, los resultados (generales) de esta lectura re-performativa habrían sido casi idénticos a los que tenían lugar en

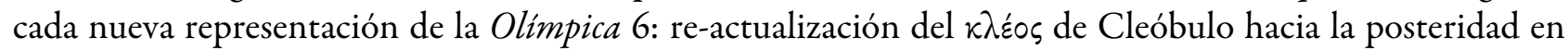
tanto buen adivino y bueno para combatir con la lanza.

Llegados a este punto, es pertinente considerar de modo un tanto más detallado los posibles contextos y modalidades puntuales de performance y re-performance para la Olimpica 6 y algunas de las estrategias que facilitan la re-lectura de $C E G 519$.

La oda dedicada a Hagesias presenta claramente dos locaciones geográficas vinculadas con la vida y la trayectoria de este laudandus: Siracusa, donde Hagesias detenta su poderío a la sombra del tirano Hierón, y Estínfalo, donde aún viven sus parientes maternos y donde quizás él mismo nació y vivió un tiempo considerable antes de trasladarse a Sicilia. La existencia de estas dos locaciones geográficas en el marco de la Olimpica 6 ha llevado a la crítica a postular dos alternativas posibles de performance para la oda: a) premier en Estínfalo y re-performance en Siracusa (cfr. Gildersleeve, 1890, p. 71; Nagy, 1990, p. 113; Hubbard, 1992, p. 80; Athanassaki, 2012, p. 134; Stamatopoulou 2014); b) premier en Siracusa y re-performance en Estínfalo (Morrison, 2007, pp. 76-79). La segunda de estas alternativas parece ser la menos probable, sobre todo si se considera el contenido de los vv. 97-105 de la oda: 


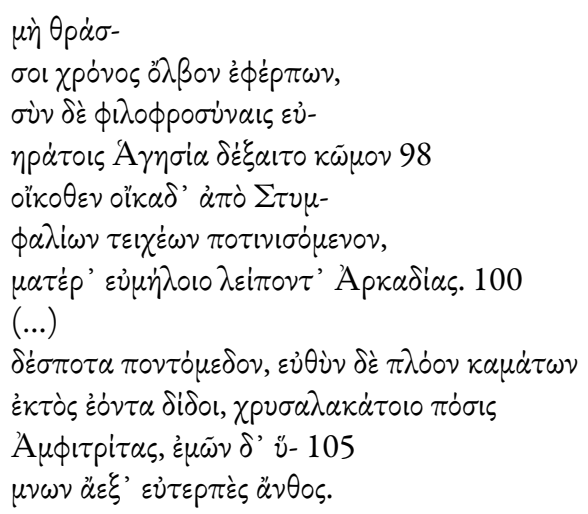

Ojalá el tiempo que avanza no dificulte su dicha y reciba con amables bondades el cortejo de Hagesias que retorna a su casa desde su casa, desde los muros estinfalios, abandonando a la madre de Arcadia de buenos rebaños (...) ¡Soberano señor del mar, otorga un viaje directo, estando sin esfuerzos, esposo de Anfitrite de huso dorado y acrecienta la alegre flor de mis himnos!

En efecto, los versos anteriores hacen pensar en una primera representación en Arcadia y en una segunda representación en Siracusa luego de una travesía marítima favorable. Puesto que la oda construye

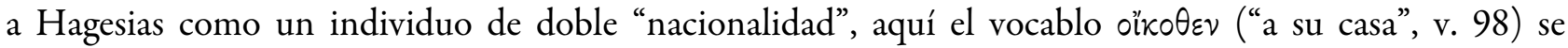
encontraría haciendo referencia al lugar al que el laudandus se dirige o regresa ( $\pi \circ \tau \iota v i \sigma o ́ \mu \varepsilon v o v, v .99)$, esto es, Siracusa, en oposición al lugar del que se va, expresado mediante el sintagma oik $\alpha \delta$ ' $a$ iò $\Sigma \tau \nu \mu \phi \alpha \lambda i \omega \nu$

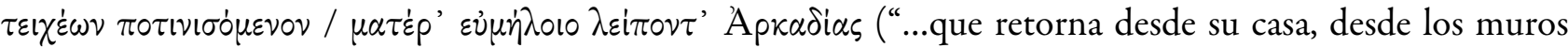
estinfalios, abandonando a la madre de Arcadia de buenos rebaños”). Asimismo, el laudandus no emprende la travesía solo, sino que lo hace en compañía de un $\kappa \tilde{\omega} \mu o s$, dejando en claro que el cortejo de victoria también se presentará en Siracusa. A este respecto, es pertinente recordar que Hagesias ya había sido descripto previamente en el v. 18 como "señor siracusano del cortejo" ( $\kappa \dot{\omega} \mu \circ v \delta \varepsilon \sigma \pi \delta \dot{\tau} \tau$. $\Sigma v p \alpha \kappa o \sigma i \omega)$.

Otro aspecto importante de esta interpretación atañe al rol de Hierón en el supuesto regreso de Hagesias. Los vv. 97-98 se colocan inmediatamente después del elogio del tirano en la oda, circunstancia que tiñe de

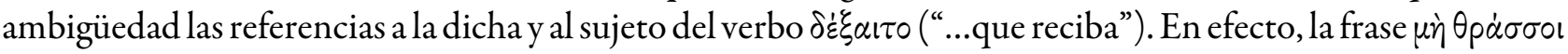

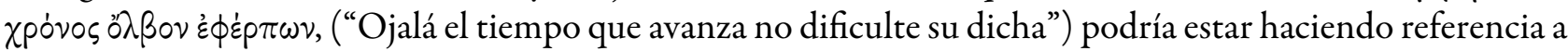
la dicha de Hierón más que a la de Hagesias, sobre todo teniendo en cuenta que el elogio previo se centra en el éxito cívico-político de este tirano como fundador de la ciudad de Etna y en su rol público como sacerdote de Deméter. A su vez, en este contexto, es bastante más plausible interpretar a Hierón y no al sustantivo abstracto

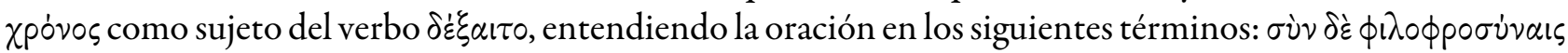

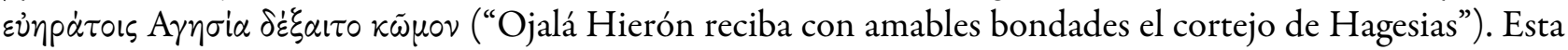
circunstancia permite generar un cuadro en el que Hierón, acaso oteando el horizonte marino en busca de señales de la nave de Hagesias, se prepara en Siracusa para recibir la celebración procedente de Estínfalo. Asimismo, dicho cuadro parecería coincidir con el precedente mítico-histórico previamente trazado en la oda, conforme al cual Hierón/Adrasto echaría de menos a Hagesias/ Anfiarao, ojo de su ejército, buen adivino y bueno para combatir con la lanza.

Más allá del contenido aquí analizado, es imposible tener absoluta certeza de la cronología de representación de la Olímpica 6. Lo que a mi juicio sí resulta indudable es que esta oda tuvo, efectivamente, por lo menos dos instancias performativas (una en Estínfalo y otra en Siracusa), como lo demuestran las estrategias compositivas que contribuyen a acomodar la oda tanto a una audiencia siciliana como a una

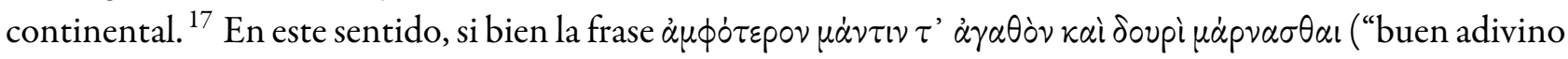
y a la vez bueno para combatir con la lanza") y las estrategias de retardo, anticipación y contextualización que la rodean (cfr. sección 2) surten sus efectos frente a ambas audiencias, acaso su importancia en tanto sintagma para vehiculizar el $\kappa \lambda \varepsilon ́ o \varsigma$ de Hagesias alcanza una dimensión máxima recién durante la (re)-performance 
siracusana, en tanto esta isla resulta el escenario en el que el laudandus finalmente pudo aunar sus habilidades mánticas y bélicas al servicio de un soberano del calibre de Hierón. Asimismo, no es difícil imaginar posteriores representaciones de la oda en Sicilia, acaso en simposios o festivales donde se conmemoraran nuevamente los éxitos de Hierón y de Cromio, por ejemplo (cfr. n. 10).

Con respecto a la modalidad performativa (solista o coral) que habría tenido la oda en su premier y posterior representación, nuevamente existen más dudas que certezas. ${ }^{18}$ La pieza deja constancia de la existencia de un $\kappa \tilde{\omega} \mu \rho \varsigma$ (cortejo) que se traslada de Arcadia a Siracusa, encabezado por Hagesias. Este cortejo bien pudo haberla representado, junto con acompañamiento musical y bailable, o simplemente haber acompañado una ejecución llevada a cabo por otro(s) individuo(s). Estas circunstancias se complejizan a partir de la mención del supuesto chorodidáskalos del epinicio, Eneas, a quien el poeta ubica en un ambiente marcadamente estinfalio: ${ }^{19}$

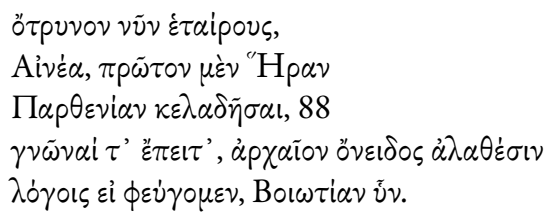

Ahora, Eneas incita a tus compañeros primero a celebrar a Hera Partenia y después a pensar si escapamos al antiguo reproche de cerda beocia con palabras verdaderas (vv. 87-90).

A partir de los versos anteriores surgen algunos interrogantes: ¿son estos $\dot{\tau} \tau \alpha i \rho o l$ (compañeros) los mismos

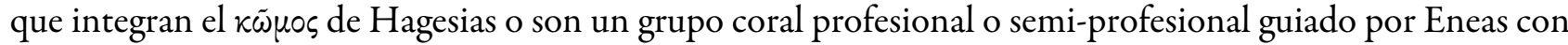
el encargo de ejecutar la Olímpica 6 en Estínfalo? ¿La celebración a Hera Partenia hace referencia a la Olimpica o constituye un canto aparte? ¿Se trasladó este mismo grupo a Siracusa para la segunda performance de la oda?Con respecto a la primera de estas preguntas, parece más probable que los $\dot{\tau} \tau \alpha i p o$

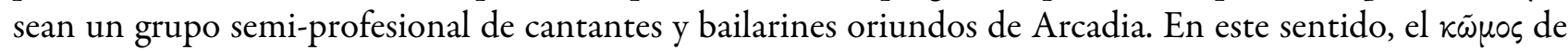
Hagesias debe entenderse en la oda como un grupo ad hoc que complementaría la ejecución coral oficial del epinicio (por lo menos en Estínfalo). Ahora bien, la posibilidad de una primera representación coral descansa, en gran medida, en la interpretación que se le dé a la primera construcción de infinitivo regida por

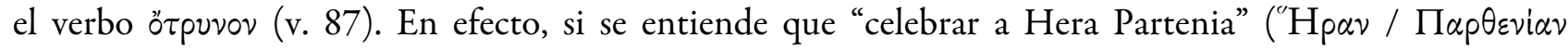
$\kappa \varepsilon \lambda \alpha \delta \tilde{\eta} \sigma \alpha l)$ es equivalente a "representar la Olimpica 6", entonces no quedarían mayores dudas acerca del carácter coral de la premier. El problema con este razonamiento es que la referencia a Hera Partenia se da solo en este verso y, más allá de ser una divinidad con un culto importante en la región de Arcadia, no se menciona de manera directa en ningún otro lugar de la oda. ${ }^{20}$ Too (1991, p. 261) ha intentado salvar esta dificultad a partir de la identificación de ciertas instancias del mito principal de la oda con la trayectoria mítica de la propia Hera en Arcadia. En este sentido, este crítico llama la atención acerca de la coincidencia

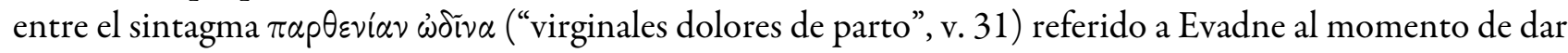

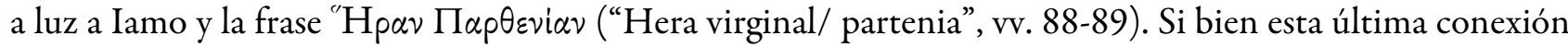
puede llegar a parecer un tanto lábil, lo cierto es que Píndaro a menudo aúna en sus composiciones el elogio y la celebración de dioses, héroes y hombres. ${ }^{21}$ En este sentido, y tomando en consideración los argumentos de Too, la Olímpica 6 bien puede ser una oda dirigida tanto a Hagesias como a Hera Partenia, especialmente si se la considera desde el punto de vista de una audiencia estinfalia. Asimismo, la utilización de los adverbios $\pi \rho \tilde{\omega} \tau 0 \nu . .$. है $\pi \varepsilon i \tau$ ' ("primero...después”) parecería crear un cuadro en el que, luego de ejecutar la performance coral de la oda=celebrar a Hera Partenia, el poeta invita al coro a reflexionar acerca de la

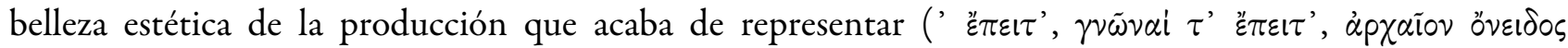

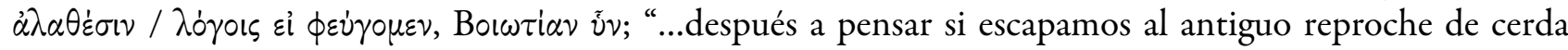
beocia con palabras verdaderas", vv. 89-90).

Con respecto al traslado de Eneas y de su grupo coral a Siracusa, esta es una cuestión que resulta imposible determinar con la evidencia con la que contamos hoy día. Por supuesto no es inverosímil que este 
chorodidáskalos se haya embarcado con sus compañeros y con Hagesias para ejecutar la oda en Sicilia una vez más. Tampoco se puede descartar que Hierón haya financiado un coro de origen siciliano para representar la Olimpica luego del retorno del "ojo de su ejército".

La re-performance del contenido de $C E G 519$ sin duda revistió un carácter mucho más informal que la doble representación coral arcadio-siracusana de la Olimpica 6. En efecto, cualquier transeúnte que circulara por las inmediaciones del epitafio de Cléobulo podría haberlo leído en voz alta, haciendo las posibilidades de reactualización de la fama del adivino-guerrero virtualmente infinitas. Para lograr este objetivo, el conjunto monumental integrado por lápida y epigrama debía desplegar determinadas "estrategias persuasivas" que atrajeran a potenciales lectores y acaso a acompañantes iletrados que desearan oír el contenido de la inscripción. En el caso de $C E G 519$, sin duda el primer y mayor atractivo que captaría la atención de quienes circulaban por la zona habría sido el bajo relieve del águila aprisionando entre sus garras una serpiente, tanto por su originalidad como por su clara remisión al universo de la mántica (cfr. sección 2). ${ }^{22}$ Este bajo relieve enmarca el contenido de la inscripción de manera clara y ordenada, otra gran ventaja a la hora de enfrascarse en su lectura. En efecto, antes del bajo relieve, en el arquitrabe, es posible leer en grandes caracteres

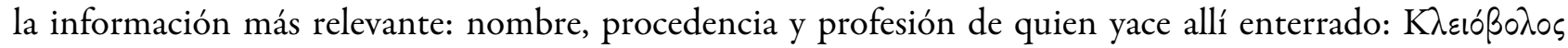

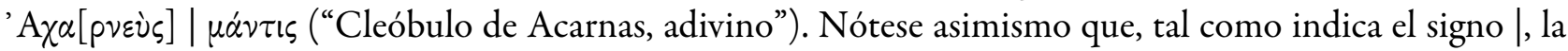
palabra $\mu \alpha \dot{\alpha} \tau$ เ s se ubica en posición central en el renglón posterior, destacándose de este modo la importancia de la profesión del individuo fallecido y anticipándose el contenido del epigrama. A continuación del bajo relieve figura el epigrama en caracteres un tanto menores. El estado de corrupción en el que ha llegado hasta nuestros días quizás pueda dar falsas impresiones acerca de la dificultad o inviabilidad de su lectura. Sin embargo, lo cierto es que la inscripción fue grabada siguiendo un patrón stoichedon con claras separaciones entre los distintos hexámetros que la integran, tal que cada uno de estos se despliega en un renglón diferente, como lo indica, una vez más, el signo | al final de verso:

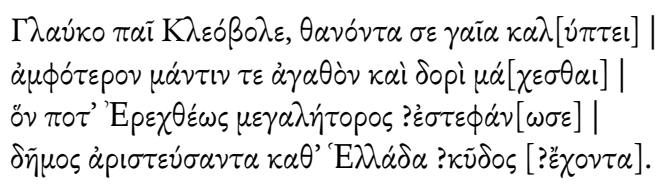

Esta prolija disposición del contenido hexamétrico, junto con la inmediata visualización frontal de todo el epigrama en la superficie de la estela, sin duda habría contribuido grandemente a facilitar su lectura.

Una vez que el transeúnte, movido por su curiosidad, decidiera acometer la lectura del epigrama, esta le depararía agradables sorpresas, sobre todo si se trataba de un individuo mínimamente versado en la tradición literaria precedente. Así, luego de detenerse en el nombre y ascendencia de Cleóbulo, y acaso pasando por

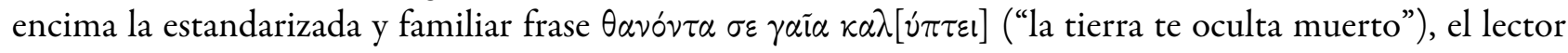

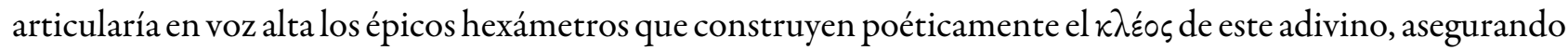
que su $\mu \nu \tilde{\eta} \mu \alpha$ permanezca vivo, salvaguardado por la estela y por la voz de inquisitivos visitantes.

\section{Conclusión}

En el presente trabajo se ha analizado la adaptación y re-utilización de la frase ả $\mu \phi o ́ \tau \varepsilon p o \nu ~ \mu \alpha \dot{\nu} \tau \tau \nu \tau \varepsilon \dot{\alpha} \gamma \alpha \theta \dot{\nu} \nu$

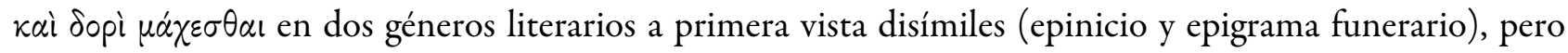
emparentados a partir de las estrategias laudatorias de corte épico dirigidas al vencedor Hagesias y al difunto Cleóbulo en tanto adivinos y guerreros y a partir de la capacidad re-performativa de inscripción y oda para

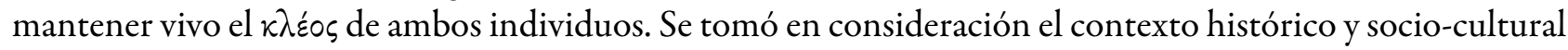
más amplio al que remite la frase, así como su función en la estructura general de oda y epigrama.

En última instancia, la utilización de la frase en los géneros analizados permite arrojar luz acerca de las estrategias y técnicas compositivas que los atraviesan. Así, el epinicio pindárico emplea el sintagma dentro 
de un excursus mitológico no solo para trazar el característico paralelismo entre laudandus y héroe mítico, sino también para balancear el elogio de Hagesias y del tirano Hierón en el marco de una secuencia que juega con las expectativas de la audiencia de la oda. Con respecto al epigrama, la aparición de la frase, inserta en una composición que exhibe claras deudas con el género épico, permite arrojar un poco más de luz acerca de los modos en los que componían los epigramatistas, tema sobre el que existen más dudas que certezas. Así, si se asume que la familia del difunto no tuvo un rol protagónico en la composición del epigrama, entonces su autor habría sido un conocedor bastante experto del acervo literario precedente con el que podía articular de manera exitosa el elogio de Cleóbulo y habría contado, quizás, con una colección propia de

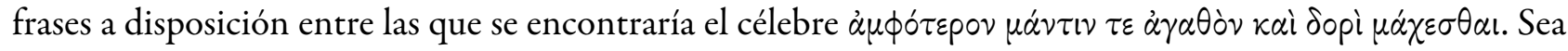
como fuere, la reaparición del sintagma empleado para elogiar a Hagesias en una composición escrita un siglo después constituye un valioso testimonio de la capacidad mito-poética de la literatura griega para reinventarse a sí misma, adquiriendo significados adicionales a medida que sus motivos se transmiten a través del tiempo y del espacio.

\section{REFERENCIAS}

Adorjáni, Z. (2014). Pindars sechste olympische Siegesode: Text, Einleitung und Kommentar. Leiden/Boston: Brill.

Allen, T. (Ed.). (1912). Homeri Opera. Oxford: Clarendon Press.

Athanassaki, L. (2012). Performance and Re-performance: The Siphnian Treasury Evoked (Pindar's Pythian 6, Olympian 2 and Isthmian 2). En P. Ágocs, C. Carey, R. Rawles (Eds.), Reading the Victory Ode (pp. 134-157). Cambridge: Cambridge University Press.

Benveniste, E. (1973). Indo- European Language and Society. Miami: University of Miami Press.

Bernabé, A. (Ed). (1996). Poetae Epici Graeci. Testimonia et fragmenta, Pars I. Leipzig: Teubner.

Bing, P. (2009). The Scroll and the Marble: studies in reading and reception in Hellenistic poetry. Michigan: Michigan University Press.

Burnett, A. (1989). Performing Pindar's Odes. CP, 84 (4), 283-293.

Carey, C. (1989). The Performance of the Victory Ode. AJP, 110 (4), 545-565.

Carey, C. (1991). The Victory Ode in Performance: The Case for the Chorus. CP, 86 (3), 192-200.

Currie, B. (2004). Reperformance scenarios for Pindar's odes. En C. Mackie (Ed.), Oral performance and its Context (pp. 49-70). Leiden/Boston: Brill.

Currie, B. (2017). Festival, Symposium, and Epinician (re) performance: The Case of Nemean 4 and Others. En R., Hunter, A. Uhlig (Eds.), Imagining Reperformance in Ancient Culture: Studies in the Traditions of Drama and Lyric (pp. 187-208). Cambridge: Cambridge University Press.

Davies, M. (Ed.). (1988). Epicorum Graecorum Fragmenta. Göttingen: Vandenhoeck \& Ruprecht.

Day, J. (1989). Rituals in stone: early Greek grave epigrams and monuments. JHS, 109, 16-28.

Day, J. (2010). Archaic Greek Epigram and Dedication: Representation and Reperformance. Cambridge: Cambridge University Press.

Daux, G. (1958). Notes de lecture. BCH, 82, 358-367.

Erbse, H. (1999). Über Pindars Umgang mit dem Mythos. Hermes 127, 13-32.

Flower, M. A. (2008). The Seer in Ancient Greece. Berkeley: University of California Press.

Foster, M. (2013). Hagesias as Sunoikistêr: Seercraft and Colonial Ideology in Pindar's Sixth Olympian Ode. CA, 32 (2), 283-321.

Gildersleeve, B. (1890). Pindar. Olympian and Pythian Odes. New York: Harper and Brothers.

Hansen, P. (Ed.). (1983-1989). Carmina epigraphica graeca saeculorum VIII-V a. Chr.n. [vol. I]... saeculi IV a. Chr.n. [II]. «Texte und Kommentare». Berlin: De Gruyter.

Heath, M. (1988). Receiving the komos: The Context and Performance of Epinician. AJP, 109 (2), 180-195. 
Heath, M. y Lefkowitz, M. (1991). Epinician Performance. CP, 86 (3), 173-191.

Hornblower, S. (2004). Thucydides and Pindar: Historical Narrative and the World of Epinikian Poetry. Oxford: Oxford University Press.

Hunter, R. y Uhlig, A. (Eds.). (2017). Imagining Reperformance in Ancient Culture: Studies in the Traditions of Drama and Lyric. Cambridge: Cambridge University Press.

Hutchinson, G.O. (2001). Greek Lyric Poetry: A Commentary on Selected Larger Pieces. Oxford: Oxford University Press.

Kurke, L. (1998). The Economy of kudos. En C. Dougherty, L. Kurke (Eds.), Cultural Poetics in Archaic Greece (pp. 131-163). Oxford: Oxford University Press.

Nagy, G. (1990). Pindar's Homer: The Lyric Possession of an Epic Past. Baltimore/London: The John Hopkins University Press.

Malkin, I. (1987). Religion and Colonization in Ancient Greece. Leiden: Brill.

Morrison, A. (2007). Performances and Audiences in Pindar's Sicilian Victory Odes. BICS, Suppl. 95, iii-146.

Morrison, A. (2010). Aeginetan Odes, Re-performance and Pindaric Intertextuality. En D. Fearn (Ed.), Aegina: Contexts for Choral Lyric Poetry. Myth, History and Identity in the Fifth Century BC (pp. 227-256). Oxford: Oxford University Press.

Morrison, A. (2011). Pindar and the Aeginetan pátrai: Pindar's intersecting audiences. En, L. Athanassaki, E. Bowie (Eds.),Archaic and Classical Choral Song: Performance, Politics and Dissemination (pp. 311-335). Berlin/Boston: De Gruyter.

Morrison, A. (2012). Performance, re-performance and Pindar's audiences. En P. Ágocs, C. Carey, R. Rawles (Eds.), Reading the Victory Ode (pp. 111-133). Cambridge: Cambridge University Press.

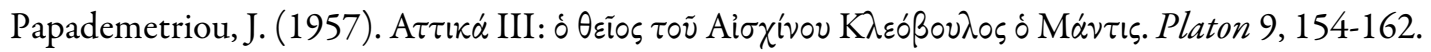

Robert, C. (1915). Oidipus: Geschichte eines poetischen Stoffs im griechischen Altertum, vols. 1-2. Berlin: Weidmann.

Slater, W. J. (1969). Futures in Pindar. CQ, 19 (1), 86-94.

Slater, W. J. (1969). Lexicon to Pindar. Berlin: De Gruyter.

Snell, B. y Maehler, H. (Eds.). (1987). Pindarus. Pars I. Epinicia. Leipzig: Teubner.

Stamatopoulou, Z. (2014). Inscribing Performances in Pindar's “Olympian 6”. TAPhA, 144 (1), 1-17.

Svenbro, J. (1988). Phrasikleia. Anthropologie de la lecture en Grèce ancienne. Paris: La Découverte.

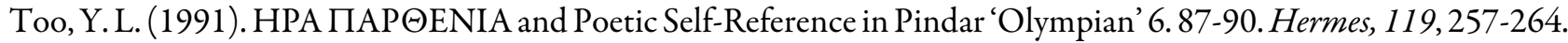

Tsagalis, C. (2008). Inscribing sorrow: Fourth-Century Attic Funerary Epigrams. Berlin/New York: De Gruyter.

von Wilamowitz- Moellendorf, U. (1886). Isyllos von Epidauros. Berlin: Weidmann.

Woodhead, A.G. (Ed.). (1959). Supplementum Epigraphicum Graecum. Leiden: Brill.

\section{Notas}

1 La edición de la Ilíada corresponde a Allen (1912).

2 La mayor dificultad para datar la oda estriba en el hecho de que las victorias olímpicas en la carrera de mulas no se encuentran listadas en $P$. Oxy. 222, acaso por el corto tiempo en el que se practicó esta disciplina. De este modo, se impone la necesidad de buscar termini ante y post quem para datar la Olimpica 6. El terminus ante quem sería la fecha de la muerte de Hierón (467/466 a.C.), fecha que ha motivado la datación de la oda en el 468 a.C. Por su parte, el terminus post quem lo constituye la fundación de la ciudad de Etna en 476/475 a.C., ya que en la oda se incluyen menciones de Hierón y de Zeus etneo (vv. 93-96). Este último terminus ha llevado a la crítica a proponer la datación alternativa 472 a.C. (cfr. Adorjáni, 2014, pp. 52-55).

3 El empleo del término $\sigma$ vขoเı $\sigma \tau \eta \dot{p}$ ha despertado las inquietudes de la crítica con respecto a su correcta interpretación. A partir de las hipótesis de los escoliastas al texto pindárico $\left(\sum 6.8 \mathrm{a}-8 \mathrm{~b}\right)$, los trabajos actuales optan por interpretar el vocablo a) como un título hereditario que evocaría el rol jugado por los Iamidas en la fundación original de Siracusa a cargo de Arquias (cfr. Hornblower, 2004, p. 185), b) como un indicador de la participación de Hagesias en las actividades 
coloniales contemporáneas de los tiranos dinoménidas, Gelón y Hierón (cfr. Malkin, 1987,pp. 93-97). De más está decir que ambas interpretaciones no resultan necesariamente excluyentes. Con respecto a los vínculos entre el rol del adivino y del colonizador en esta Olimpica, véase Foster (2013).

4 La edición corresponde a Snell-Maehler (1987) y las traducciones son propias.

5 Otras odas en las que Píndaro emplea secuencias míticas que tienen a Anfiarao y a Adrasto como protagonistas son $P$. 8.38-56; N. 9.7-28, en menor medida I. 7.10-12,31-36.

6 En la versión que figura en $S E G$ XVI 193 se imprime la variante $\mu \alpha[\chi \eta \tau \eta \dot{\eta}]$. La única letra claramente visible es la $\mu$. Se

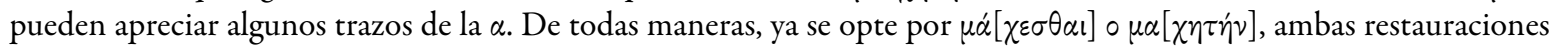
son sumamente convincentes dado el contexto inmediato.

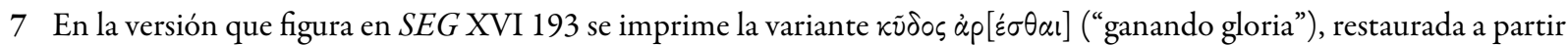
de Iliada 18.293-294. El sintagma es, de todas maneras, ilegible y ninguna de las dos conjeturas puede afirmarse con certeza. Cfr. Daux (1958, p. 365), quien, a propósito de estos suplementos, dice categóricamente: “je ne lis rien de sûr".

8 Para ejemplos de epigramas arcaicos y clásicos dedicados a guerreros caídos en batalla, cfr. entre otros $C E G 2,4,5,6,12$; 13; 19; 27; 47; 82; 83; 87; 99; 102; 112; 118; 136; 142; 145; 155, SEG LVI 40, XLVIII 83, IG 7.53. Para un análisis complementario de este epigrama a partir de sus estrategias poéticas arcaizantes y de sus diferencias con los ejemplares del siglo IV a.C., cfr. Tsagalis (2008, pp. 3-9; 230-232; 262-263).

9 Papadametriou (1957, pp. 160) fue el primero en observar la coincidencia entre ambas frases.

10 Foster (2013, pp. 288-289) cree poder estrechar aún más las similitudes entre estas dos figuras a partir de la presencia

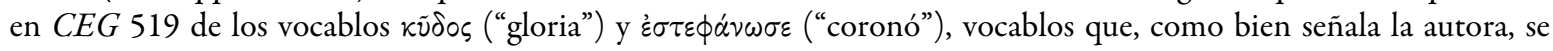
encuentran íntimamente relacionados con los atributos y el entorno del atleta pindárico. El problema del razonamiento de Foster estriba en el hecho de que coloca mucho peso interpretativo en dos palabras ( $\kappa \tilde{v} \delta \circ \varsigma$ y $\dot{\varepsilon} \sigma \tau \phi \phi \dot{\alpha} \nu \omega \sigma \varepsilon)$ que son prácticamente ilegibles en el epigrama. Para la definición de $\kappa \tilde{\delta} \delta \varsigma$, cfr. Benveniste (1973, p. 348). Para su interpretación y aplicación en el campo de las lides atléticas, véase Kurke (1998).

11 En este sentido, Hagesias habría cumplido un rol parecido al del siracusano Cromio, pariente de Hierón, al que se le dedican las Nemeas 1 y 9 , en donde abundan menciones tanto de su maestría atlética como militar. Para Foster (2013) la alusión al desempeño militar de Hagesias no debe entenderse solo de modo literal, sino también interpretarse en términos de roles político-cívicos colaborativos entre Hierón y este laudandus: "Pindar transfers the camaraderie between a military mantis and his commander in war to Hagesias and Hieron's own civic collaboration, (p. 301)”.

12 Para un análisis exhaustivo de las funciones cumplidas por los adivinos en el marco de enfrentamientos bélicos y los distintos patrones de comportamiento que exhibían en relación con los jefes militares a los que debían responder, véase Flower (2008, pp. 153-187). A menudo este vínculo oscilaba entre la comprensión y entendimiento mutuo y la animadversión total y completa entre ambas partes.

13 Por más apropiado que pueda resultar para la presente argumentación, la frase $\sigma \tau p \alpha \tau i \tilde{\alpha} \varsigma$ ỏ $\phi \theta \alpha \lambda \mu \grave{\nu} \nu$ parece no relacionarse de modo directo con las habilidades adivinatorias de Hagesias en el campo de batalla, sino más bien con su desempeño satisfactorio general en el enfrentamiento. En este sentido, ỏ $\phi \lambda \lambda \lambda \mu$ ó debería entenderse como "pride, crown" (cfr. Slater s.v. c), teniendo la frase el significado "orgullo o corona de mi ejército". Así figura en Olímpica 2.10, por ejemplo. Sin embargo, dada la habilidad pindárica para aunar múltiples significados en un solo sintagma, quizás no sea pertinente apresurarse a descartar el significado más específico de ỏ $\phi \theta \lambda \lambda \mu$ ó en relación con la práctica adivinatoria. Los dos sentidos aquí descriptos bien pueden estar presentes en la frase.

14 Foster (2013, 300-301) aplica un razonamiento semejante para efectuar la identificación Hagesias-Anfiarao, HierónAdrasto, pero su argumento se centra en el vínculo cívico-político entre Hagesias y Hierón, a partir de la caracterización del primero como adivino y co-fundador, no en el vínculo bélico. Para el elogio de Hierón dentro de la Olímpica 6, cfr. también vv. 93-96. Para los distintos espacios en los que se articula la oda, véase la sección 3 del presente trabajo.

15 La bibliografía sobre el fenómeno de re-performance en la oda pindárica es vasta. Véanse principalmente los trabajos de Currie $(2004 ; 2017)$ y de Morrison $(2007 ; 2010 ; 2011 ; 2012)$. El pasaje pindárico más visitado a este respecto es $N$. 4.13-16, en donde el poeta declara que si el padre del vencedor aún continuara con vida a menudo ejecutaría la oda en su cítara. Para un estudio de este fenómeno y de su importancia en distintos géneros literarios de la antigüedad, consúltese Hunter-Uhlig (2017).

16 La tesis de Day ha encontrado en Bing (2009) uno de sus mayores detractores. Una de las principales objeciones de este crítico es el bajo grado de alfabetismo en la sociedad griega de los siglos VI-V a.C. Sin embargo, tal como plantea Day (2010, pp. 30-32), la postura de Bing es producto de un pensamiento dicotómico del tipo "todos o ninguno". Por supuesto que no todos los individuos querían o podían leer las inscripciones, pero que ninguno lo hiciera resulta igualmente imposible. En el medio de este espectro existían, por ejemplo, estrategias de lectura parcial, llevadas a cabo por personas semi-letradas. Asimismo, con el correr de los años, el número de individuos capaces de acometer la lectura de los epigramas habría ido aumentando. En el rango temporal en el que se ha colocado a $C E G 519$ (375-350 a.C.) sin duda el nivel de alfabetismo sería más alto que en los siglos anteriores. 
17 Para un análisis pormenorizado de todas estas estrategias, cfr. Stamatopoulou (2014). Para la propuesta de una performance enteramente siracusana que interpreta el escenario arcadio en términos ficticios, ver Erbse (1999, pp. 16-18).

18 Para el debate inicial acerca de si las odas de Píndaro eran representadas por un coro o por un solista, véanse los trabajos de Heath (1988) y Heath-Lefkowitz (1991) a favor de la postura solista y los trabajos de Burnett (1989) y Carey (1989; 1991) a favor de la postura coral. A partir del análisis del corpus pindárico, parece imposible hoy en día optar de manera general por alguna de estas dos opciones. Se impone, en cambio, la necesidad de considerar que distintos epinicios hayan presentado en su premier distintas modalidades performativas (corales o solistas), de acuerdo con los deseos del vencedor y/o otras coyunturas particulares.

19 A juzgar por la mención de los parientes maternos oriundos de Estínfalo (v. 77) y de los vínculos entre Eneas y su coro con la región de Arcadia, este personaje bien pudo haber sido un familiar de Hagesias por el lado materno.

20 Esto ya fue señalado por Slater (1969) y Heath-Lefkowitz (1991, p. 197).

21 Los ejemplos de esta unión en el corpus pindárico son muy abundantes. Como instancia más representativa de esta tendencia, remito al comienzo de la Olimpica 2, encabezado por la triple serie interrogativa A $\nu \alpha \xi ı \phi o ́ p \mu l \gamma \gamma \varepsilon \varsigma$ vँ $\mu \nu 0 \mathrm{l}, / \tau i \nu \alpha$

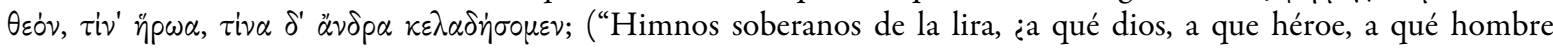
celebraremos?").

22 A este respecto, es necesario recordar que, si bien hoy en día las estelas aparecen grises y descoloridas frente a nuestros ojos, los bajos relieves y, en algunos casos, los trazos de las letras, se pintaban con tintes diversos, de entre los que sobresalían especialmente el rojo y el azul. El colorido del complejo habría aportado una estrategia adicional para captar la atención de los transeúntes. 